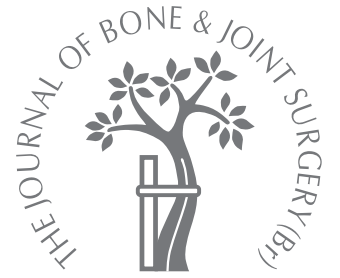

K. Hayashi,

A. Fotovati,

S. A. Ali,

K. Oda,

H. Oida,

M. Naito

From Hara Doi

Hospital, Fukuoka, Japan

\title{
Prostaglandin EP4 receptor agonist augments fixation of hydroxyapatite-coated implants in a rat model of osteoporosis
}

The reduced stability of hydroxyapatite (HA)-coated implants in osteopenic conditions is considered to be a major problem. We therefore developed a model of a boosted cementless implantation in osteopenic rats.

Twelve-week-old rats were either ovariectomised (OVX) or sham-operated (SO), and after 24 weeks plain or HA-coated implants were inserted. They were treated with either a prostaglandin EP4 receptor agonist (ONO-4819) or saline for one month.

The EP4 agonist considerably improved the osteoporosis in the OVX group. Ultrastructural analysis and mechanical testing showed an improvement in the implantbone attachment in the HA-coated implants, which was further enhanced by the EP4 agonist. Although the stability of the HA-coated implants in the saline-treated OVX rats was less than in the SO normal rats, the administration of the EP4 agonist significantly compensated for this shortage. Our results showed that the osteogenic effect of the EP4 agonist augmented the osteoconductivity of HA and significantly improved the stability of the implant-bone attachment in the osteoporotic rat model.

It is estimated that the worldwide incidence of hip fractures will rise to 6.26 million per year by 2050 compared with 1.66 million in $1990 .^{1}$ In most developed countries there has been an increase in the elderly population, some of whom will undergo total hip arthroplasty (THA).

Many of these patients will have osteoporosis and therefore the development of a hip replacement which will confer stability of the implant in osteopenic conditions is necessary.

Cementless arthroplasty using biomaterials such as hydroxyapatite (HA) is increasingly attracting more attention. ${ }^{2-6}$ Over the past 20 years, we have investigated cementless alternatives for hip arthroplasty, using osteoconductive biomaterials such as HA in various animal models including the rat and the dog. ${ }^{7-11}$

The results of in vivo animal studies have suggested that there are potential benefits from HA coating in orthopaedic applications in men. Furthermore, the excellent clinical results of HA-coated THA have proved the usefulness of HA coating for cementless THA. ${ }^{5,12,13}$ All of our animal studies have been in models with normal bone without osteoporosis. ${ }^{3-11}$ The major problem for elderly patients with severe osteoporosis is a weak implant-bone attachment which limits the use of HA-coated cementless THA. ${ }^{14}$
In our previous studies, we have used ovariectomised (OVX) and denervated rats as animal models for osteoporosis and have shown the positive osteoconductive effect of HAcoated implants. ${ }^{14-17}$ However, the implantbone attachment strength of HA-coated implants in OVX rats was still significantly lower than that in normal control rats. ${ }^{14}$ In this study, therefore, we investigated whether the additional use of an osteogenic agent might improve the osteoconductive effect of HA.

Prostaglandins (PGs) are a group of lipid mediators involved in the maintenance of local homeostasis. Of the several types of PG, PGE 2 has the most well-known effect on bone metabolism ${ }^{18-20}$ by induction of both osteoblastogenesis and osteoclastogenesis and the integration of the two. Agonists of these receptors have been suggested for clinical use. Unfortunately, the general stimulation of $\mathrm{PGE}_{2}$ does not only affect bone metabolism. The use of a non-selective agonist for $\mathrm{PGE}_{2}$ is limited by side-effects including the induction of pain, gastrointestinal complications, hypotension and abnormality of contractility of the uterus. ${ }^{21,22}$

$\mathrm{PGE}_{2}$ exerts its effects through interaction with G-protein-coupled cell-surface receptors ${ }^{23}$ including EP1, EP2, EP3 and EP4. EP4 is considered to be the most potent and specific 


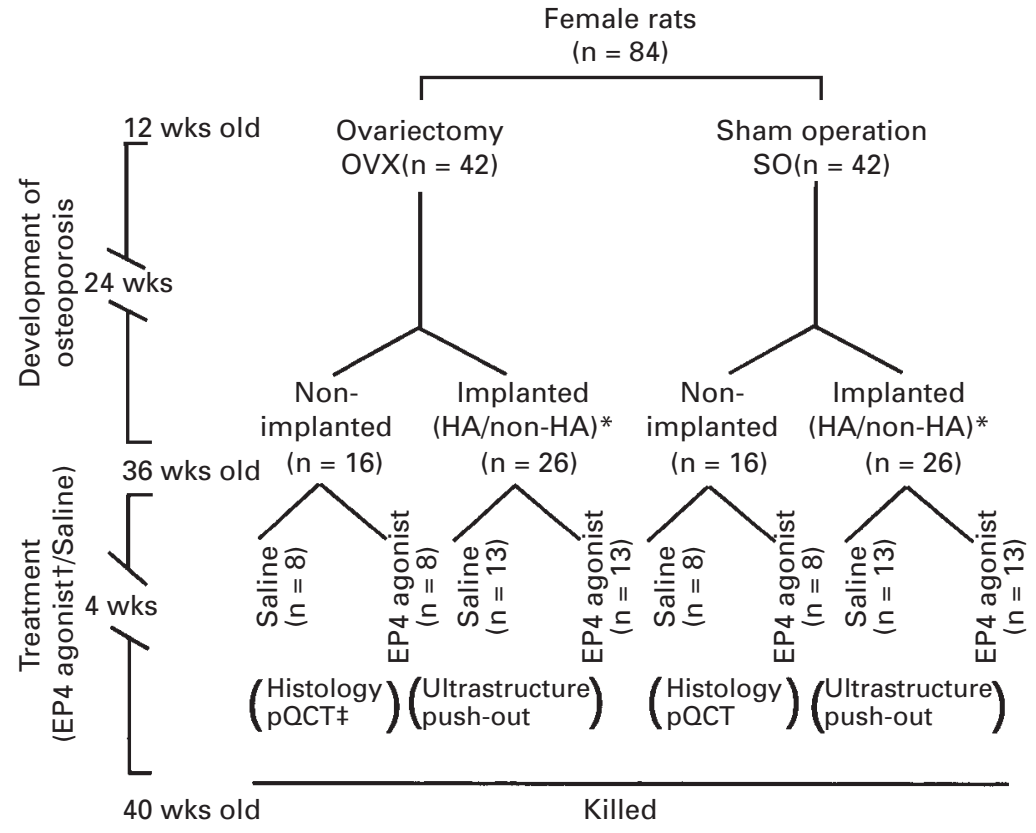

Fig. 1

Diagram showing the experimental plan and the number of animals chosen for each group ( ${ }^{*} \mathrm{HA}$, hydroxyapatite-coated, lateral (right/left) femur; non-HA, nonhydroxyapatite coated, co-lateral (left/right) femur; $\dagger$ ONO 4819 as EP4 agonist $15 \mathrm{mg} / \mathrm{kg} / \mathrm{twice}$ daily starting immediately after implantation; $\ddagger$ pQCT, peripheral qualitative $\mathrm{CT}$ ) receptor for mediating anabolic action in bone. Induction of the osteogenic effect by $\mathrm{PGE}_{2}$ requires a concentration 100 times greater than that for specific $\mathrm{PGE}_{2}$-EP4 agonist stimulation. ${ }^{21}$ Therefore, for prevention of the unwanted effects of high concentrations of $\mathrm{PGE}_{2}$, EP4-selective agonists are expected to be more practical for clinical use. ${ }^{18}$

\section{Materials and Methods}

Animal experimental design and surgical procedures. All the animal experimental procedures were approved by the Hospital and University animal experimentation committees. We used 84 mature Charles Wistar female rats of mean weight 246.45 (SD 52.82) which were purchased from Seac Co Ltd (Fukuoka, Japan). They were divided randomly into two experimental groups so that both had the same mean weight (Fig. 1). The rats were from the same stock and selection was done completely blindly adjusting only for the mean weight of each individual group. All the rats were maintained in separate plastic cages with 12-hour light, and 12-hour dark cycles. They had free access to food and water. After one week of acclimatisation, at the age of 12 weeks, one group ( $\mathrm{n}=42$ ) was bilaterally ovariectomised. The other group $(\mathrm{n}=42)$ was sham-operated (SO) and comprised the control group.

Twenty-four weeks later the femora of 26 rats from each group, i.e. a total of 52 femora were implanted with an HAcoated prosthesis on one side and a non-coated implant on the other. The prostheses were $23 \mathrm{~mm}$ long and $1.4 \mathrm{~mm}$ in diameter, both cylindrical with the same surface roughness. The HA coating was deposited using a plasma-spray technique, as previously described. ${ }^{14}$ The thickness of the coating was $20 \mu \mathrm{m}$ and the crystallinity was $55 \%$ with the amorphous layer composed of calcium phosphate. The $\mathrm{Ca} / \mathrm{P}$ ratio was 1.66 . The mean surface roughness of the
HA-coated and the non-coated implants was $4.3 \mu \mathrm{m}$ (SD 0.6 ) and $4.3 \mu \mathrm{m}$ (SD 0.5), respectively. All the implants had been sterilised in an autoclave which did not affect the HA coating.

Immediately after implantation, both the OVX and SO groups were further divided into EP4-agonist and salinetreated subgroups, with 13 rats in each subgroup. They were injected subcutaneously, twice a day with $15 \mu \mathrm{g} / \mathrm{kg}$ body-weight of EP4 agonist dissolved in saline, or with saline only, for four weeks starting from the day of implantation. The dosage was chosen according to previous studies. ${ }^{18-20}$

The remaining rats (16 rats in the OVX and SO groups) did not have implants and were also treated similarly with either EP4 agonist or saline (eight rats for each subgroup) starting from the same time as the implanted rats. The femora of these rats were used for measurement of the bone mineral density (BMD) and for histological studies.

All the rats were killed after treatment for four weeks by an overdosage of anaesthetic and their femora were excised. Thirty-six of the implanted femora from each group were used for measurement of implant-bone strength. Sixteen implanted femora from each group were used for ultrastructural studies of implant-bone interaction. Twenty-four non-implanted femora were used for measurement of the BMD and eight for histological studies.

Bone mineral density. BMD was measured using peripheral quantitative CT (pQCT) on the non-implanted femora of the rats from each experimental group as previously described. ${ }^{18}$

Histological examination. For light microscopy, the femoral samples were fixed in $10 \%$ phosphate-buffered formalin for 48 hours. They were decalcified using $10 \%$ formic acid. Decalcification was continued by regular exchange of the 


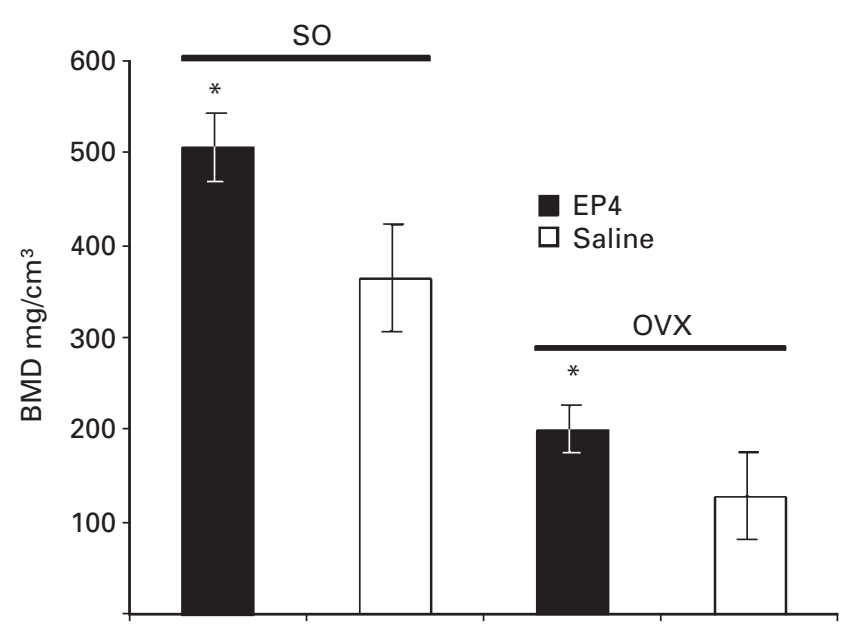

Fig. 2

Bar chart showing the BMD of the trabecular bone of femora measured by pQCT in the drug-treated and the saline-treated groups, respectively ( $n=$ 8) (* significantly different compared with the related drug-treated group; drug versus saline for non-OVX (OVX-) $(\mathrm{p}<0.01)$; drug versus saline for OVX $(O V X+)(p<0.05))$. OVX, ovariectomised; SO, sham-operated. acid until complete softening of the femora was achieved (one to two weeks). The femora were then washed in running water to remove any residual formic acid. The decalcified bones were dehydrated in gradients of alcohol $(70 \%$ to $100 \%)$ followed by xylene and then embedded in paraffin wax. After dehydration and rehydration the sections were stained with haematoxylin and eosin according to the standard protocol.

Ultrastructural studies. The ultrastructural profile of the implant-bone interface was observed by back-scattered SEM (JSM - 840 A, JEOL Co. Ltd, Tokyo, Japan). The femora containing the implants were embedded in resin and were cut using a diamond saw. Images of the implantbone ultrastructural profile were photographed using a Polaroid instant camera (Polaroid Corporation, USA). Image analysis. Histological and ultrastructural profiles of bones obtained from the experimental groups were further studied by image analysis. Briefly, the density of bone formation was measured after colour binarisation of the osseous trabeculae in the histological and electron micrographs using a WinROOF 5.03 image analyser
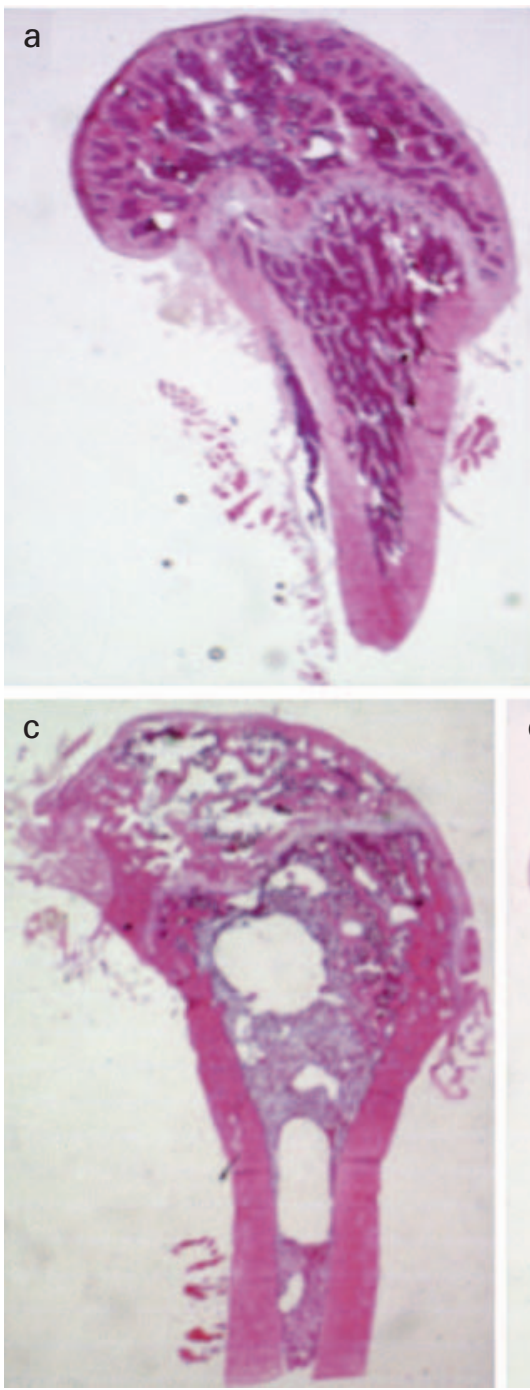
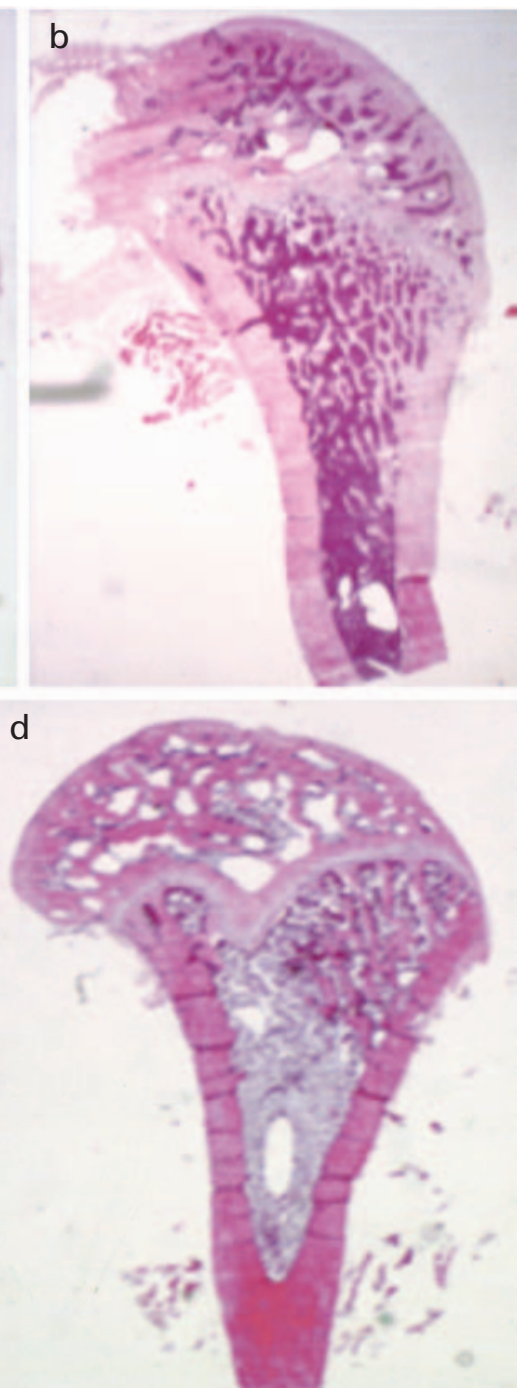

Fig. 3

Light microscopy showing sections in the mid-sagittal plane of a) sham-operated saline-treated, b) sham-operated EP4-agonist-treated, c) overiectomised salinetreated and d) overiectomised EP4-agonist-treated femora (haematoxylin \& eosin $\times 5$ ). 

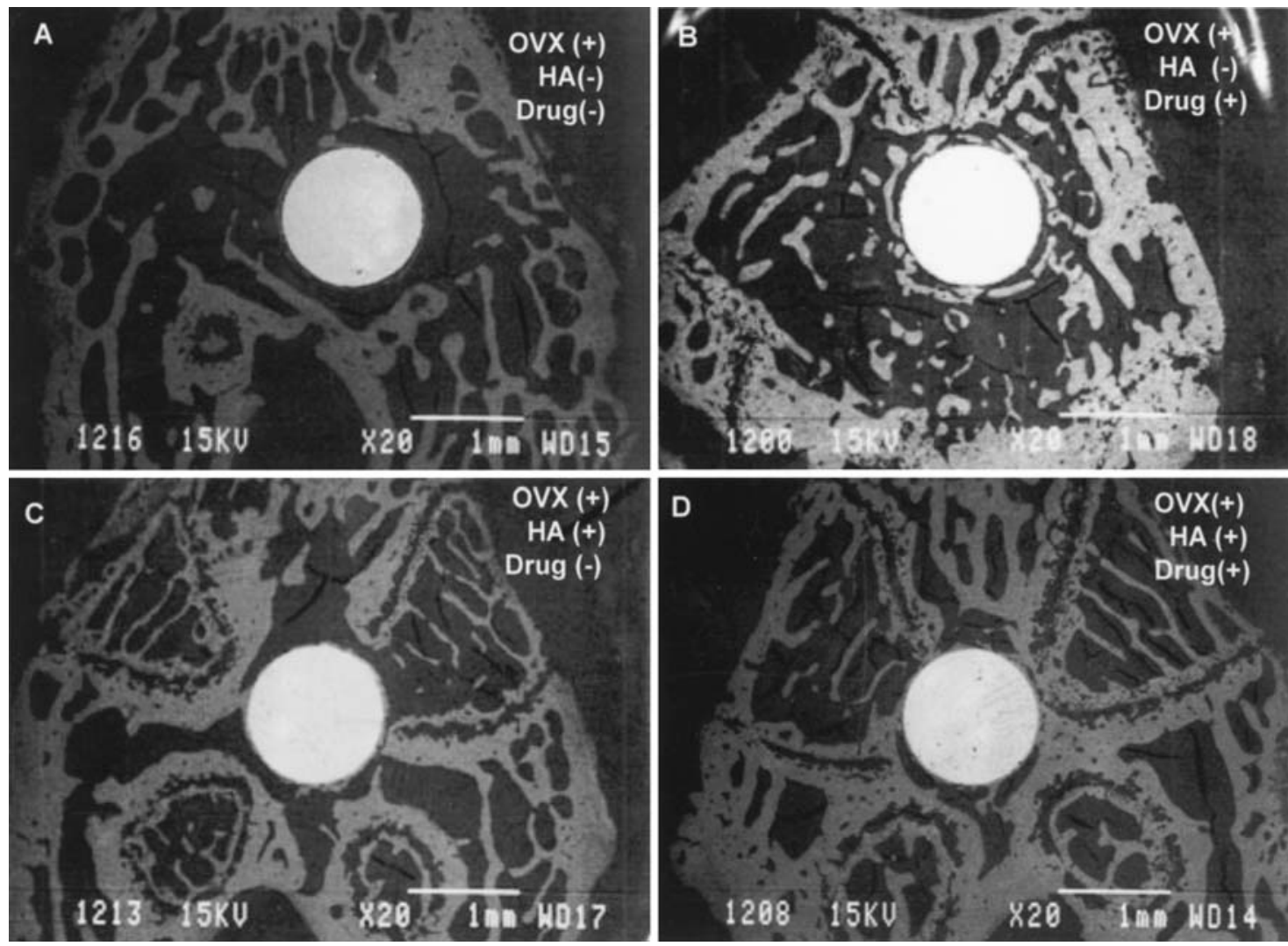

Fig. 4

Ultrastructural study of implanted femora in the ovariectomised group showing the interaction between osteoconductivity of hydroxyapatite (HA)- and EP4-agonist-induced osteogenesis. The upper row shows this interaction in non-HA implants and the lower row that in HA-coated implants. The left column is after saline treatment and the right column shows the interaction after treatment with agonist (see text).

(Mitani Corporation, Tokyo, Japan). The mean density of the distal part of the femora containing the trabecular light microscopy and osteogenic network surrounding the implants (electron microscopy) was calculated as a ratio of the osseous to the non-osseous area, i.e. the intratrabecular spaces.

Bone-implant attachment strength. This was measured by pushing-out the implants as previously described. ${ }^{14}$ The energy necessary for breaking the implant-bone interaction and pushing out the implant was termed 'the bone-implant attachment strength'. This was shown by a sharp fall in pressure. The peaks were measured and recorded.

Statistical analysis. The results of each group of animals were calculated as the mean (SEM). The difference among groups was assessed by two-way ANOVA and followed by Fisher PLSD test for post hoc comparison, for assessment of the difference between all pairs of HA-coated and noncoated, and EP4-agonist and saline-treated animals in order to evaluate the effect of the HA coating and administration of the EP4 agonist, and their combination. We considered a $p$ value of less than 0.05 to be significant. The data were analysed using JMP 5.1 Statistical Discovery Software (SAS Institute Inc, Cary, North Carolina).

\section{Results}

Bone mineral density. BMD of the femora was reduced significantly in the OVX compared with the SO group ( $\mathrm{p}<$ 0.05 ; Fig. 2). The OVX group treated with saline showed the lowest level of BMD $129.3 \mathrm{mg} / \mathrm{cm}^{3}$ (SD 46.8). The administration of EP4 agonist significantly increased the BMD in the OVX group $200.0 \mathrm{mg} / \mathrm{cm}^{3}$ (SD 28.6; $\mathrm{p}<0.05$ ). However, in spite of this improvement in the EP4-agonist-treated OVX group it was still significantly lower than that in the salinetreated $\mathrm{SO}$ rats $365.5 \mathrm{mg} / \mathrm{cm}^{3}$ (SD 57.2; $\mathrm{p}<0.001$ ). In the control group, administration of agonist resulted in a further increase of BMD $506.3 \mathrm{mg} / \mathrm{cm}^{3}$ (SD 36.3) compared with those treated with saline only $(\mathrm{p}<0.01)$.

Histological findings (Fig. 3). The bone structure of the distal femur in the SO group showed a normal profile (Fig. 3A) but there was an extensive loss of trabecular bone in the OVX group (Fig. 3C) as was evidenced by bone-depleted spongy structures. However, administration of agonist in the OVX group gave some evidence of ossification in the area which had undergone heavy bone loss (Fig. 3D). The histological profile of the femora in the SO rats did not show a dramatic change after administration of the agonist (Fig. 3B). 


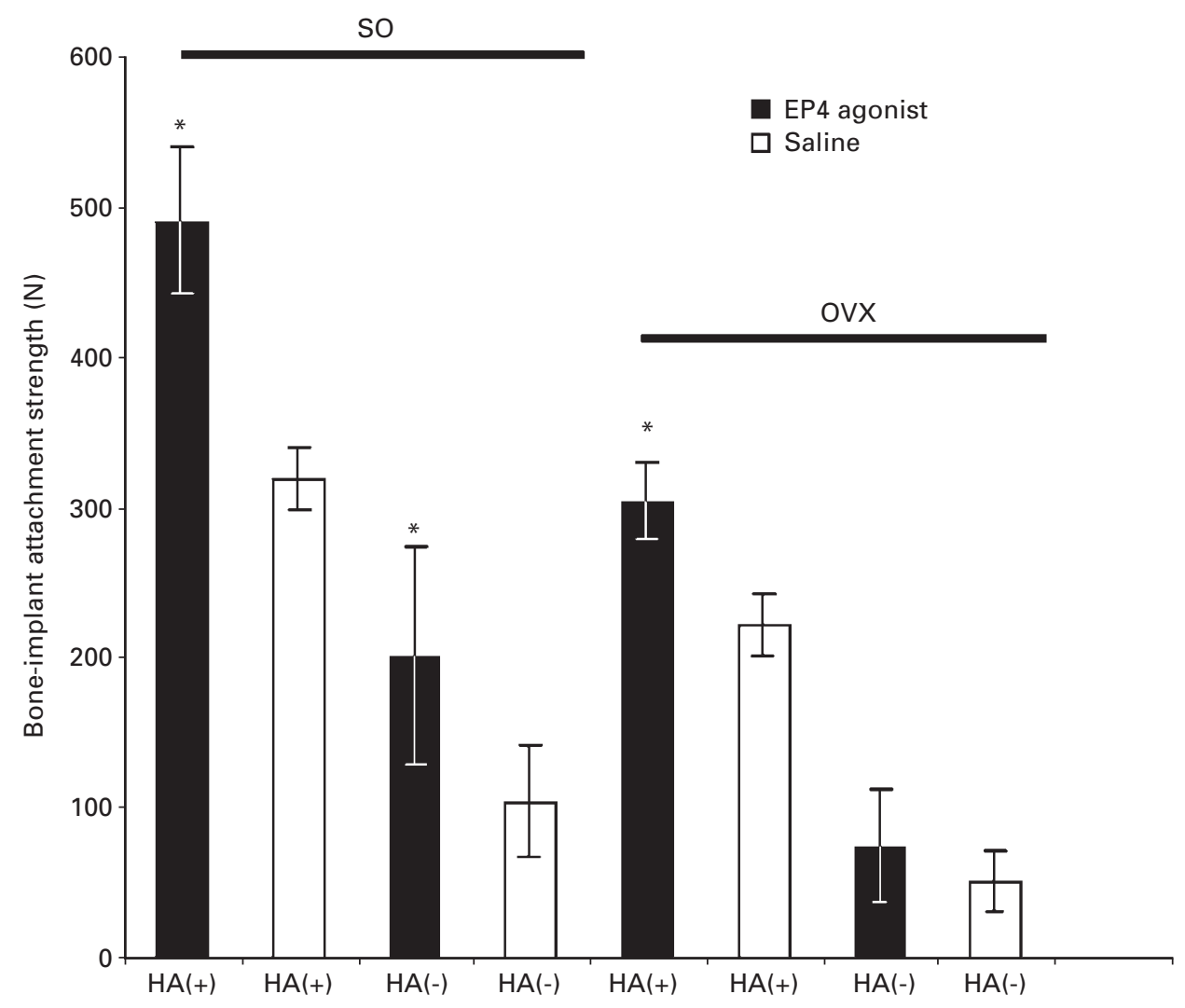

Fig. 5

Bar chart showing the bone-implant attachment strength. The implants were pushed out of the femora and the strength was measured in Newtons (N). The solid and open bars show the drug-treated and the saline-treated groups, respectively $(n=8)(*$ significantly different compared with the related drug-treated group: drug versus saline for sham-operated (SO), hydroxyapatite (HA) ( $p<0.05)$; drug versus saline for SO, non-HA $(p<0.05)$; drug versus saline for ovariectomised (OVX), HA $(p<0.01)$; and drug versus saline for OVX, non-HA $(p=0.20))$.

Image analysis showed the ratio of the trabecular osseous area to the non-osseous area in SO agonist-treated animals $(72.6 \%$, graded as I) to be higher than that in both saline and agonist-treated OVX groups, and slightly higher than in the SO saline-treated group $(63.0 \%$, graded as II). Among OVX animals the agonist-treated group had a higher osseous to non-osseous ratio $(53.5 \%$, graded as III), comparing with saline-treated OVX animals which showed the lowest ratio compared with all other groups $(44.2 \%$, graded as IV).

Ultrastructural analysis. The profile of the OVX group is shown in Figure 4. There was an obvious lack of bone contact around non-HA implants (Figs 4A and 4B). This was more obvious in the saline-treated OVX group (Fig. 4A), but a bony network developed around the HA implants (Figs 4C and 4D). The bony network was further expanded in HA implants of agonist-treated rats (Fig 4D). The combination of drug and HA coating established the most extensive network of bone interaction around the implant (Fig. 4D).

Image analysis of the ultrastructural profile of implantbone interaction showed the lowest density of bone around
non-HA implants in the saline-treated group. The highest density was seen in HA implants in the agonist-treated rats, $30.2 \%$ and $45.8 \%$, respectively. The density of bone around the HA implant in the saline-treated group (34.9\%) and non-HA implants in the agonist-treated group $(35.3 \%)$ was almost the same. Therefore, the histological profile of the bone network around the implants was graded as high (grade I) in HA-coated implants of agonist-treated rats, medium (grade II), in both HA implants in saline-treated and non-HA implants in agonist-treated groups and low, (grade III) in non-HA implants in saline-treated rats.

Bone-implant attachment strength. The attachment strength was lower in OVX compared with SO animals and in the saline group compared with the agonist-treated group (Fig. 5). The non-HA implants in OVX rats which only received saline showed the lowest strength $51.0 \mathrm{~N}$ (SD 20.5). The strongest was in the HA-coated implants in non-OVX healthy rats which had received the agonist $489.8 \mathrm{~N}$ (SD 50.1). Implant-bone attachment strength in OVX, salinetreated animals was significantly increased by using HAcoated implants $220.9 \mathrm{~N}$ (SD 21.2), compared with non-HA implants $(p<0.001)$. However, it was still significantly 
lower than the same subgroup, i.e. HA-coated, salinetreated $\mathrm{SO}$ rats $303.5 \mathrm{~N}$ (SD 25.8), at a mean of approximately $30 \%$ less; $\mathrm{p}<0.01$ ). Nevertheless, treatment with agonist increased the strength of HA-coated implants in the OVX group 318.1 N (SD 22.3) to a level which was not significantly different from that in the same HA-coated implants in the saline-treated SO group $(\mathrm{p}=0.35)$. The agonist treatment also significantly increased the implant-bone attachment strength in the group of normal rats with HAcoated implants compared with those treated with saline only $(\mathrm{p}<0.05)$.

\section{Discussion}

Our study has shown that a combination of the physiological, osteogenic effect of prostaglandin EP4 agonist and the osteoconductivity of HA improved the stability of bone fixation in osteopenia.

The application of growth factors, such as bone morphogenic protein and transforming growth factor- $\beta$, and also coating the implant with HA, have been suggested for improving the stability of implants in patients with osteoporosis. However, there are insufficient studies upon which to make any conclusions. ${ }^{24,25}$

There are also several agents which either activate osteoblasts or inhibit the differentiation and function of osteoclasts. $^{26}$ To date, none has been able to restore a proper balance between the formation and resorption of bone and have been mainly undertaken in normal non-osteopenic models, with controversial findings..$^{27,28}$ The use of agents which only prevent bone resorption, such as aledronate, may not be enough to confer a strong interforce and the addition of agents with an osteogenic effect may be necessary.

In our study, the OVX group showed a significant reduction in BMD, as was expected (Fig. 2). The administration of EP4 agonist improved the BMD in this group, although it did not increase it to the level seen in the normal rats (Fig. 2). This was attributed to the dosage and injection schedule of EP4 agonist in our study. We started the treatment after osteoporosis had been established unlike our previous study, in which the EP4 agonist was administered more frequently but immediately after OVX. ${ }^{18}$ The loss of BMD in the OVX group was fully restored in the subgroup which had been treated with agonist. However, the schedule of our study was designed to be more representative of hip arthroplasty in elderly patients with established osteoporosis. We speculated that even this improvement might be enough to boost the osteoconductivity of HA and thereby confer effective implant-bone stability. Additionally, there was a general improvement in the histological recovery of bone loss in the OVX group when they received the EP4 agonist (Fig. 3). Taken together, these data showed that there was some improvement in the osteogenesis of trabecular bone in the OVX group which received the agonist.

In the next step we investigated whether there was any interaction between HA and EP4-agonist-induced osteo- genesis at the implant-bone interface. Ultrastructural studies showed a considerable interaction between the HA coating and the surrounding bone (Fig. 4C), as we have previously reported. ${ }^{14}$ However, this was intensified with the addition of EP4 agonist (Fig. 4D). The ultrastructural profile appeared to show that by using agonist there was further enhancement of the interface, especially in the OVX group (Figs 4B and 4D).

Finally, we investigated whether this enhanced interaction actually improved the implant-bone attachment strength in vivo. We performed biomechanical tests to measure the attachment strength. It was observed that although implant-bone attachment of the HA-coated implants in the saline-treated OVX group was significantly stronger than in the non-HA group as we had previously reported, ${ }^{14}$ it was still significantly lower than in the HA-coated implants in saline-treated normal rats (Fig. 5). However, in our study, the use of EP4 agonist enhanced the implant-bone attachment strength of HA-coated implants of OVX rats to that of saline-treated normal rats (Fig. 5). Thus, the osteogenic effect of EP4 seemed to boost osteoconductivity of HA and compensated for the reduction of bone-implant attachment strength in OVX rats compared with normal. We found no significant difference in the implant-bone attachment of the non-HA smooth implants in OVX, with or without treatment with agonist. This suggested the importance of HA for the establishment of a strong attachment between the implant and agonist-induced newly-formed bone, especially when an implant with a smooth surface was used.

We are investigating the side-effects of EP4 agonist in a preclinical trial. If it is successful, this approach may be considered as an adjunct to cementless hip arthroplasty in elderly patients.

No benefits in any form have been received or will be received from a commercial party related directly or indirectly to the subject of this article.

\section{References}

1. Cooper C, Campion G, Melton LJ 3rd. Hip fractures in the elderly: a world-wide projection. Osteoporos Int 1992;2:285-9.

2. Bauer TW, Geesink RGT, Zimmerman R, McMahon JT. Hydroxyapatite-coated femoral stems: histological analysis of components retrieved at autopsy. J Bone Joint Surg [Am] 1991;73-A:1439-52.

3. Cook SD, Thomas KA, Kay JF, Jarcho M. Hydroxyapatite-coated titanium for orthopedic implant applications. Clin Orthop 1988;232:225-43.

4. Ducheyne P, Cuckler JM. Bioactive ceramic prosthetic coatings. Clin Orthop 1992; 276:102-14.

5. Geesink RGT, de Groot K, Klein CP. Bonding of bone to apatite-coated implants. J Bone Joint Surg [Br] 1988;70-B:17-22.

6. Søballe K, Toksvig-Larsen S, Gelineck J, et al. Migration of hydroxyapatite coated femoral prostheses: a roentgen stereophotogrammetric study. J Bone Joint Surg [Br] 1993;75-B:681-7.

7. Hayashi K, Inadome T, Mashima T, Sugioka Y. Comparison of bone-implant interface shear strength of solid hydroxyapatite and hydroxyapatite-coated titanium implants. J Biomed Mater Res 1993;27:557-63.

8. Hayashi K, Mashima T, Uenoyama K. The effect of hydroxyapatite coating on bony ingrowth into grooved titanium implants. Biomaterials 1999;20:111-19.

9. Hayashi K, Matsuguchi N, Uenoyama K, Kanemaru T, Sugioka Y. Evaluation of metal implants coated with several types of ceramics as biomaterials. J Biomed Mater Res 1989;23:1247-59.

10. Hayashi K, Uenoyama K, Matsuguchi N, Sugioka Y. Quantitative analysis of in vivo tissue responses to titanium-oxide and hydroxyapatite-coated titanium alloy. $J$ Biomed Mater Res 1991;25:515-23. 
11. Hayashi K, Inadome T, Tsumura H, Nakashima Y, Sugioka Y. Effect of surface roughness of hydroxyapatite-coated titanium on the bone-implant interface shear strength. Biomaterials 1994;15:1187-91.

12. D'Antonio JA, Capello WN, Manley MT, Geesink R. Hydroxyapatite femora stems for total hip arthroplasty: 10- to 13-year follow-up. Clin Orthop 2001;393: 101-11.

13. Hayashi K, Nakashima Y, Inadome T, Sugioka Y, Noda I. Comparison of hydroxyapatite-coated with Harris/Galante porous uncemented total hip replacement: a retrospective matched study and experimental study for the improvement of HA-coating. Bioceramics 1994;7:441-6.

14. Hara T, Hayashi K, Nakashima Y, Kanemuru T, Iwamoto $Y$. The effect of hydroxyapatite coating on the bonding of bone to titanium implants in the femoral of ovariectomised rats. J Bone Joint Surg [Br] 1999;81-B:705-9.

15. Hayashi K, Fotovati A. Animal models of osteoporosis. In: An YN, ed. Orthopaedic issues in osteoporosis. Boca Raton: CRC Press, 2003:71-89.

16. Hayashi K, Uenoyama K, Mashima T, Sugioka Y. Remodeling of bone around hydroxyapatite and titanium in experimental osteoporosis. Biomaterials 1994;15: $11-16$.

17. Hayashi K, Uenoyama K, Matsuguchi N, Nakagawa S, Sugioka Y. The affinity of bone to hydroxyapatite and alumina in experimentally induced osteoporosis. J Arthroplasty 1989;4:257-62.

18. Yoshida K, Oida H, Kobayashi T, et al. Stimulation of bone formation and prevention of bone loss by prostaglandin E EP4 receptor activation. Proc Natl Acad Sci USA 2002;99:4580-5.
19. Sasaoka R, Terai $\mathbf{H}$, Toyoda $\mathbf{H}$, et al. A prostanoid receptor EP4 agonist enhances ectopic bone formation induced by recombinant human bone morphogenetic protein2. Biochem Biophys Res Commun 2004;318:704-9.

20. Tanaka M, Sakai A, Ichida S, et al. Prostaglandin E(2) receptor (EP4) selective agonist (ON0-4819.CD) accelerates bone repair of femoral cortex after drill-hole injury associated with local up regulation of bone turnover in mature rates. Bone 2004;34: $940-8$.

21. Ke HZ, Shen VW, $\mathbf{O} \mathbf{i}$, et al. Prostaglandin E2 increases bone strength in intact rats and in ovariectomized rats with established osteopenia. Bone 1998;23:249-55.

22. Parfitt K, ed. Prostaglandins. In: Martindale: the complete drug reference. Thirty-second ed. London: Pharmaceutical Press, 1999:1414-15.

23. Narumiya S, Sugimoto Y, Ushikubi F. Prostanoid receptors: structures, properties, and functions. Physiol Rev 1999;79:1193-226.

24. Bensen CV, Schuttle HD Jr. Joint replacement in osteoporotic bone. In: An YH, ed. Orthopaedic issues in osteoporosis. Boca Raton, Florida: CRC Press, 2003:405-14.

25. Eskhoff DG. Bone ingrowth to prosthetic surfaces in osteoporotic bone. In: $\mathrm{An} \mathrm{YH,}$ ed. Orthopaedic issues in osteoporosis. Boca Raton, Florida: CRC Press, 2003:445-55.

26. Rodan GA, Martin TJ. Therapeutic approaches to bone disease. Science 2000;289: 1508-14

27. Mochida Y, Bauer TW, Akisue T, Brown PR. Alendronate does not inhibit early bone apposition to hydroxyapatite-coated total joint implants. J Bone Joint Surg [Am] 2002;84-A:226-35.

28. Skripitz R, Aspenberg P. Implant fixation enhanced by intermittent treatment with parathyroid hormone. J Bone Joint Surg [Br] 2001;83-B:437-40. 